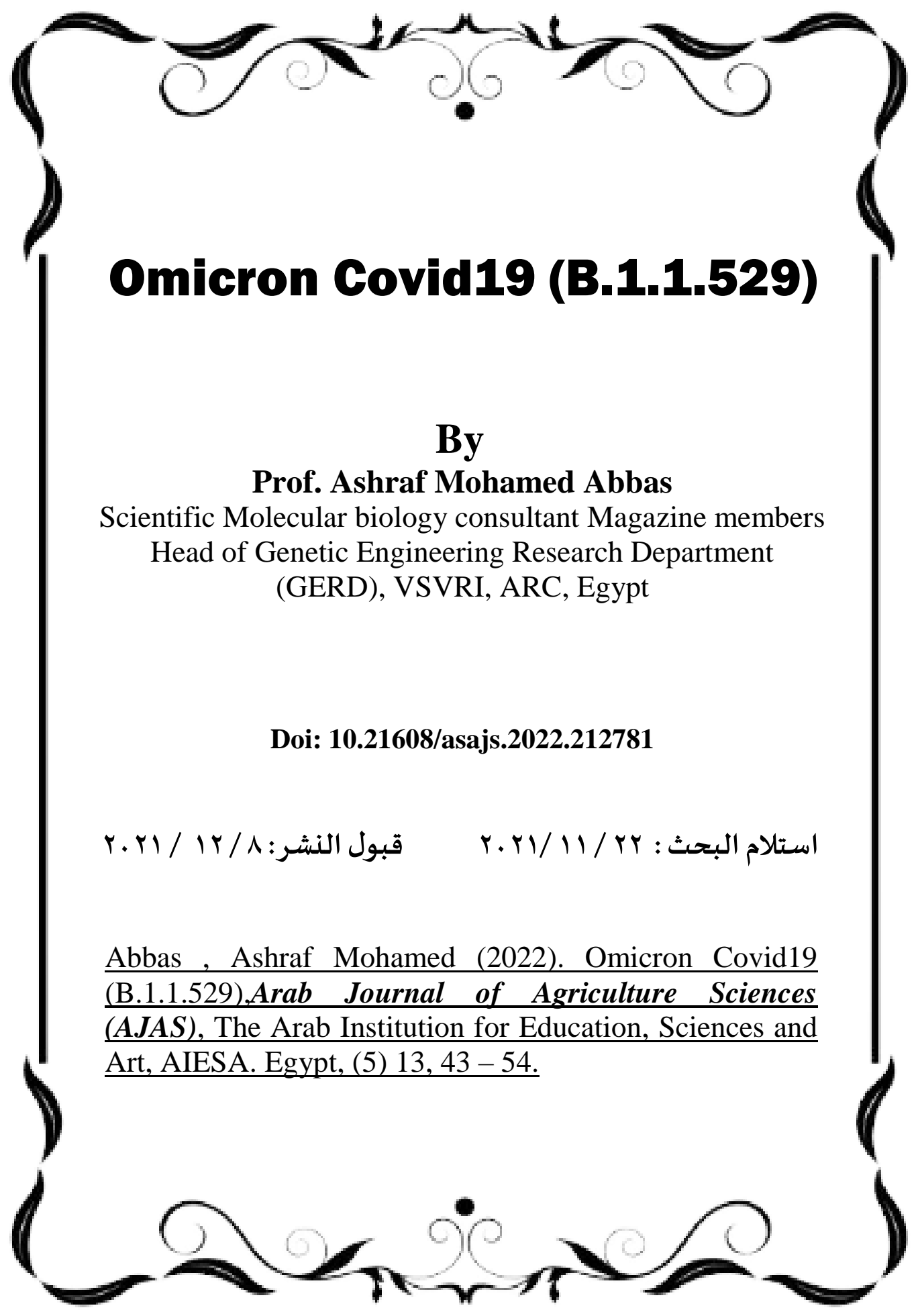




\section{Omicron Covid19 (B.1.1.529)}

\section{Abstract:}

On November $23^{\text {rd }}$, scientists in South Africa alerted the world to a new variant of SARS-CoV-2, the virus that causes COVID-19. Officially named the B.1.1.529 variant, it has been designated Omicron as part of the World Health Organization (WHO)'s Greek naming system, and has put health officials and researchers around the world on high alert as they make sense of its potential implications for the ongoing global pandemic. In addition to South Africa, the new variant has been detected in at least 15 countries in Africa, Europe, and North America, according to $C N N$, prompting myriad restrictions on international travel. The variant is likely already present in additional countries, including the United States, The Wall Street Journal reports. (1)

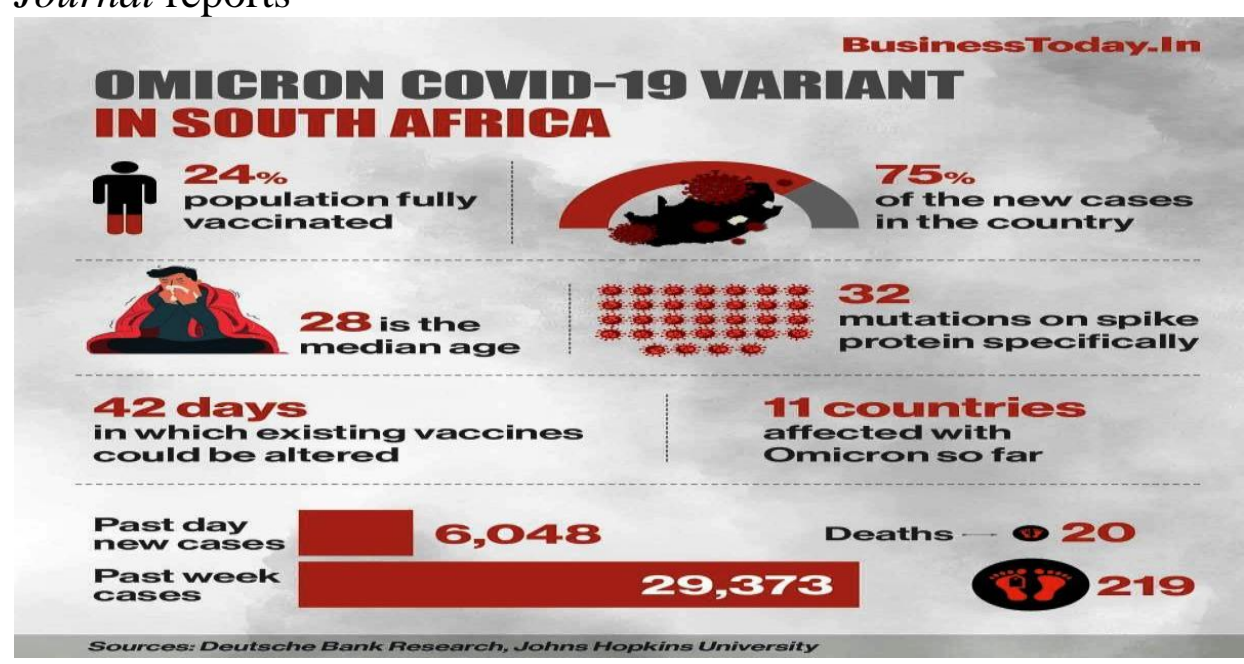

Figure (1) Omicron variant in South Africa (Deutsche Bank Research Johns Hopkins University 


\section{How contagious is Omicron?}

The Deutsche Bank report says the South African Covid19 variant could be more contagious than other variants. The reason for the widespread concern is the incredibly high number of mutations, with 32 on the spike protein specifically, which is the part of the virus that allows it to enter human cells. "That's much more than we've seen for previous variants, and raises the prospect it could be a more transmissible version of the virus, although scientists are still assessing this," the report adds. Though the WHO experts are still studying this new virus it's in the early stages, experts think there are some worrying signs. The WHO has said initial signs show the Omicron could cause "reinfection". Also, this variant has a lot more mutations than other variants had, particularly on the spike protein. "This could make this variant more infectious, and there is also the possibility that vaccines are less effective against it too," says the Deutsche Bank report.

\section{Is Omicron a deadly variant? ${ }^{[2]}$}

Experts are still finding out how deadly this virus is but the Deutsche Bank report says even if it does prove to be "less deadly", this could still be outweighed by a rise in "transmissibility", resulting in more overall hospitalizations and deaths.

"Take an example where 100 people have a virus and it sends $10 \%$ into hospital. That means 10 end up in hospital. Then let's say the virus mutates, and it then only has an 8 per cent chance of sending you to hospital but is 40 per cent more infectious. Then 140 people have the virus, but 11 end up in the hospital, which was actually more than under the previous strain, even though the hospitalization rate for each case is actually lower," the report adds. also says that "higher transmissibility" and a "lower fatality" rate doesn't necessarily lead to fewer 
deaths, since the higher transmissibility means it affects a lot more people.

\section{How many countries have reported Omicron cases?}

So far, the main cluster of this Covid strain is in South Africa, although that doesn't necessarily mean it originated there. Botswana has so far identified 19 cases of this virus. The Netherlands reported 13 cases of this variant from two flights that arrived in the country. Hong Kong had found two cases of this variant; Belgium confirmed that they had a case; and Israel's health ministry also confirmed a case. Similarly, the UK found 2, Italy one; Australia two; Germany three; and Denmark two confirmed cases of Omicron Covid-19 variant.

\section{How prevalent is the Omicron variant?}

As per the Deutsche Bank Research report, the Omicron variant, or B.1.1.529, has become the dominant strain in South Africa, with genome sequencing indicating that 75 per cent of the new cases in the country are from this variant. In Gauteng province, the figure stands at 90 per cent. The WHO has said the first documented case of this Covid-19 variant was first detected on November 9, which means the virus may be spreading for the past three weeks.

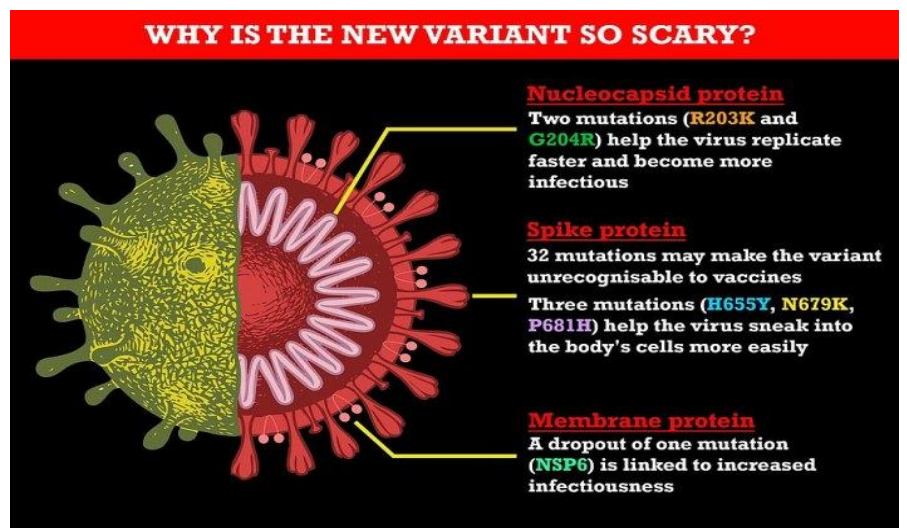

Figure (2) Why is the new Variant so scary News WWC Omicron variant

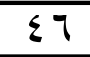


The new Covid-19 variant: B.1.1.529

More mutations may make it spread faster

Spike protein helps New variant has 32

the virus enter human mutations on the

cells spike protein

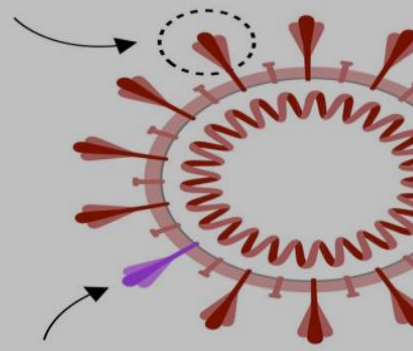

New variant has 10 mutations

on the 'receptor binding domain'

- which gains entry to cells

Source: South Africa Centre for Epidemic Response and Innovation $\quad$ BIBCC

Figure (3) The new Covid -19 variant: B.1.1.529 ${ }^{(3)}$

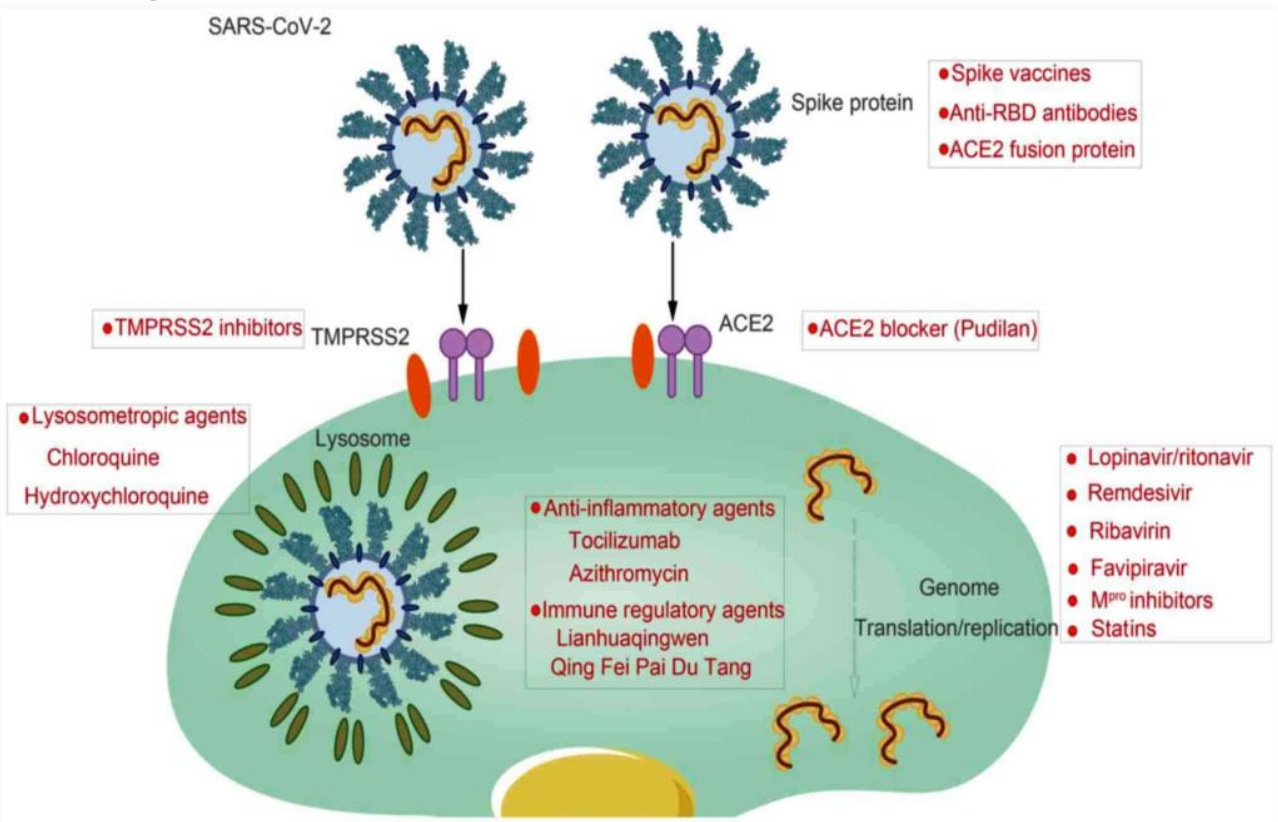

Figure. (4) Mechanism by which SARS-CoV-2 infects host cells. The $S$ protein on the surface of the SARS-CoV-2, a newly emerged pathogen spreading worldwide, binds with 
high affinity to the human ACE2 receptor to gain entry into target cells, including those of lung and gastrointestinal tissues. SARS-CoV-2 employs the serine protease TMPRSS2 for $S$ protein priming. The pharmaceutical agents which disrupt the COVID-19 infection of host cells are exhibited in red based on the mechanism and the specific site of action. SARS-CoV-2, severe acute respiratory syndrome coronavirus 2; ACE2, angiotensin-converting enzyme 2; TMPRSS2; transmembrane protease serine 2; S, spike; COVID-19, coronavirus disease 2019; RBD, receptor binding domain $^{(4) \text {. }}$

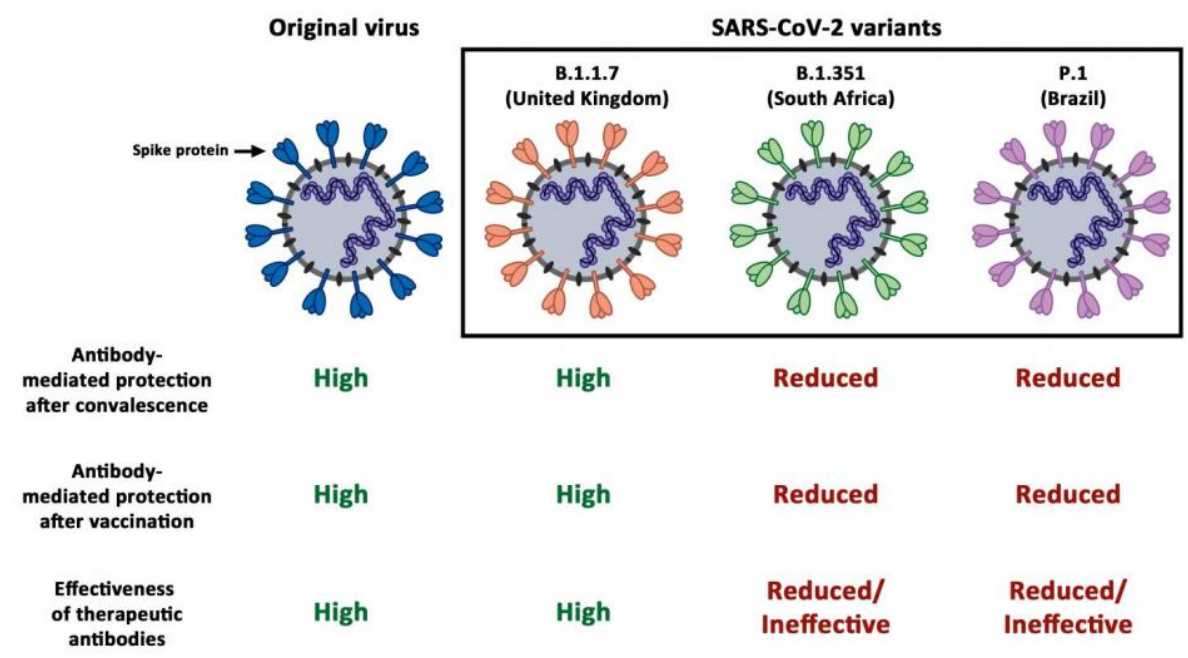

Figure (5) :-Evidence of escape of SARS-CoV-2 variant B.1.351 from natural and vaccine-induced $\operatorname{sera}^{(5)}$. 


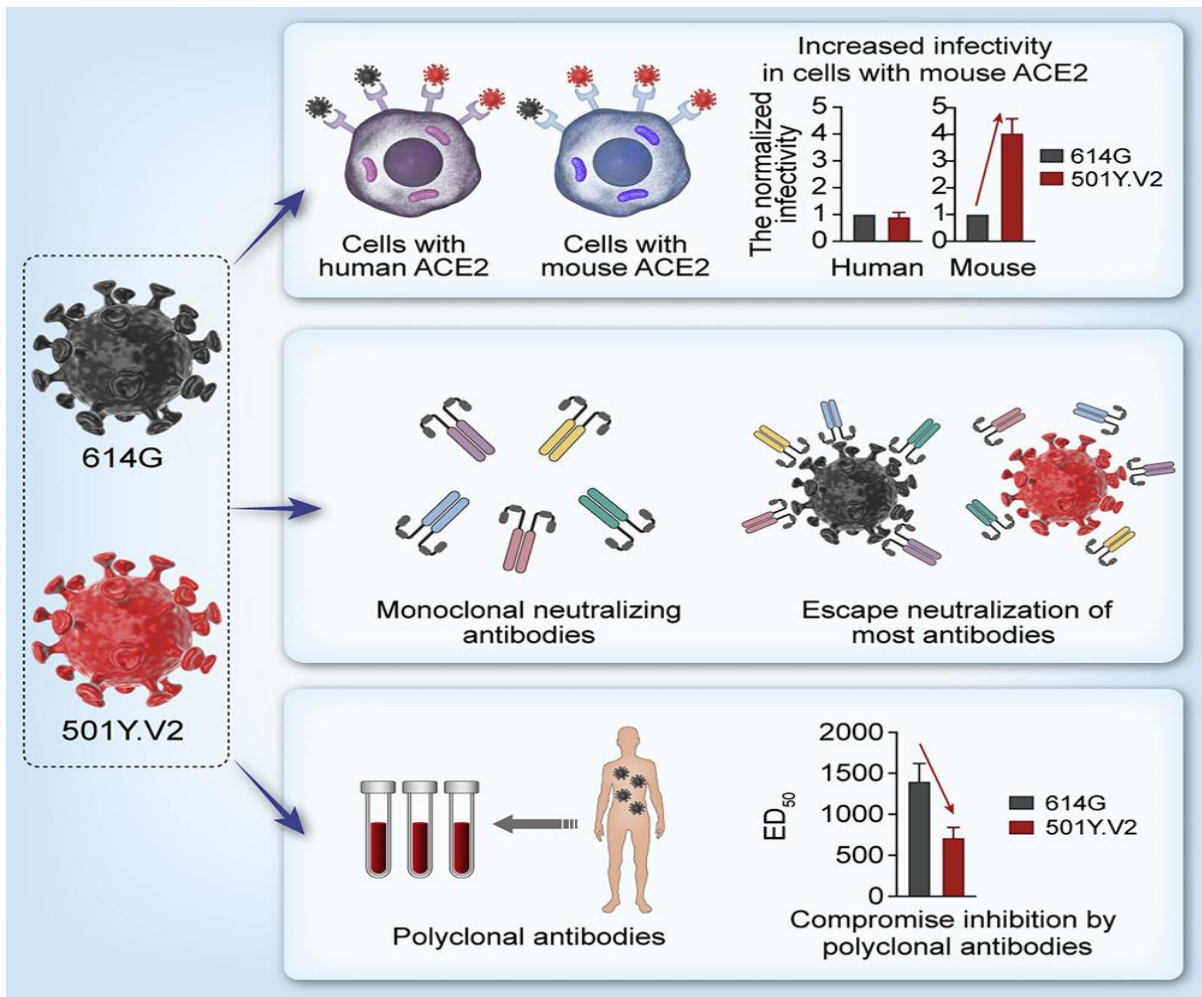

Figure (6):-SARS-CoV-2 501Y.V2 variants lack higher infectivity but do have immune escape ${ }^{[6]}$

\section{What could happen in near future?}

As per the Deutsche Bank report, a strong leading indicator will be what happens to hospitalizations and deaths in South Africa, where this has become dominant. If there is a noticeable spike, then that carries concerning implications for elsewhere.

However, much higher vaccination in Europe and the US, which could offer protection against the virus spread. Notably, only 24 per cent of the South African population is fully 
vaccinated, while the percentage is 58 per cent in the United States and 69 per cent in France. However, another concerning factor is the older population across Europe and the US, which may result in higher hospitalizations and deaths. In South Africa, the median age in the country is 28 , much lower than Western Europe's median age of 44, says the Deutsche Bank report.

\section{What are the overall implications on stock markets?}

Reports of the spread of a new COVID-19 variant of concern in Southern Africa have caused sharp negative moves in stock markets. "Market concern is understandable, in our view. Early evidence suggests a very rapid displacement of the delta variant, which is known to be dominant in South Africa," says a report by financial services major Credit Suisse. When looking at the emergence of the delta wave, the key indicator was how quickly delta increased in its share of sequenced cases. Sequencing data suggests B.1.1.529 has a different evolutionary pathway but shares a few common mutations with the C.1.2, Beta and Delta variants, the report adds.

\section{Will existing vaccines work?}

There's no clarity on whether existing vaccines will work against Omicron or not, but laboratory testing is already underway according to the South Africa National Institute for Communicable Diseases. Initial thoughts from the institute are that "partial immune escape is likely", considering numerous mutations in comparison to the sequence that existing vaccines were designed against. Experts say it could take a minimum of 6week time to develop a new vaccine against this variant. But, the riders like conducting trials could add months.

Pharmaceutical companies say they're monitoring the situation to see if modifications to their vaccines need to be made. Pfizer and Moderna previously stated that it would take about six weeks to alter their mRNA vaccines, according

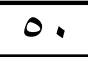


to $C N N$, and spokespeople from both companies tell The New York Times that they're investigating whether such alterations are necessary or if booster shots will fend off the Omicron variant. However, fully understanding how these and the variant's other mutations may alter the dynamics of Omicron infections will require researchers to gather and analyze a lot more data over the coming days and weeks.

In the meantime, experts emphasize that masking, washing hands, and social distancing remains helpful. "We have been through all of this before with the Alpha and Delta variant," University of Leicester clinical virologist Julian Tang tells Science Focus. "But the main difference is that the scientific evidence for the effectiveness of masks and social distancing has increased - and there is more conviction now amongst scientists that these measures do work to reduce the spread of the virus.

\section{Countries are asked to do the following:}

- Enhance surveillance and sequencing efforts to better understand circulating SARS-CoV-2 variants.

- Submit complete genome sequences and associated metadata to a publicly available database, such as GISAID.

- Report initial cases/clusters associated with VOC infection to WHO through the IHR mechanism.

- Where capacity exists and in coordination with the international community, perform field investigations and laboratory assessments to improve understanding of the potential impacts of the VOC on COVID-19 epidemiology, severity, effectiveness of public health and social measures, diagnostic methods, immune responses, antibody neutralization, or other relevant characteristics.

Individuals are reminded to take measures to reduce their risk of COVID-19, including proven public health and social measures such as wearing well-fitting masks, hand hygiene, 
physical distancing, improving ventilation of indoor spaces, avoiding crowded spaces, and getting vaccinated.

-increase in transmissibility or detrimental change in COVID-19 epidemiology; OR increase in virulence or change in clinical disease presentation; OR -decrease in effectiveness of public health and social measures or available diagnostics, vaccines, therapeutics $^{\text {(7) }}$

Author question what is the main cause of emersion of omicron Covid 19 or new variants?

- Some countries reported the administration of the fourth dose of Covid 19 vaccination.

- Level of Covid 19 vaccination in some global areas is less than $10 \%$. 


\section{References}

[1]https://www.the-scientist.com/news-opinion/omicron-iswhos-fifth-variant-of-concern-experts-urge-patience-69472.

[2] https://www.businesstoday.in/coronavirus/story/omicronvariant-how-deadly-is-the-south-african-covid-19-strain-willexisting-vaccines-work-all-you-need-to-know-313734-2021-1129.

[3] https://www.bbc.com/news/health-59520945. The new Covid -19 variant :B.1.1.529.

[4] Wang, R., X. Luo, F. Liu and S. Luo (2021). "Confronting the threat of SARS-CoV-2: Realities, challenges and therapeutic strategies." Experimental and Therapeutic Medicine 21(2): 1-1. [5] https://www.eurekalert.org/multimedia/680769). Evidence of escape of SARS-CoV-2 variant B.1.351 from natural and vaccine-induced sera.

[6] Li, Q., J. Nie, J. Wu, L. Zhang, R. Ding, H. Wang, Y. Zhang, T. Li, S. Liu and M. Zhang (2021). "SARS-CoV-2 501Y. V2 variants lack higher infectivity but do have immune escape." Cell 184(9): 2362-2371. e2369.

[7]https://www.who.int/news/item/26-11-2021-classificationof-omicron-(b.1.1.529)-sars-cov-2-variant-of-concern 
